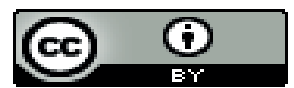

Online

\title{
Diagnosis of lymph node tuberculosis using the GeneXpert MTB/RIF in Bangladesh
}

\author{
TE Nur ${ }^{1 凶}$, AU Hosna ${ }^{2}$, N Rayhan $^{3}$, N Nazneen $^{4}$
}

\begin{abstract}
The purpose of this study was to evaluate the accuracy of the GeneXpert M. tuberculosis (MTB)/ rifampicin (RIF) test for the detection of MTB in lymph node aspirated samples. This study was conducted in the Department of Pathology, Bangabandhu Sheikh Mujib Medical University, Bangladesh. This study was done during the period from July 2013 to May 2015. A total of 317 clinically suspected tuberculous lymphadenitis patients without malignancy were included in the study. The culture test and GeneXpert test were used for detection of MTB in lymph node aspirated material. Among the 317 samples tested, the GeneXpert detected the DNA of MTB in 167 samples $(52.7 \%)$, whereas culture test was positive in $74(23.3 \%)$ specimens. GeneXpert also detected 8 RIF resistance cases. GeneXpert sensitivity and specificity results were assessed according to culture results. The sensitivity and specificity of the GeneXpert assay was $95.9 \%$ and $60.5 \%$, respectively. The implementation of the GeneXpert MTB/RIF assay may dramatically improve the rapid diagnosis of lymph node TB. The GeneXpert MTB/RIF may replace usual conventional method like culture test for detection of MTB. Key words: $M$. tuberculosis, lymph node TB, rifampicin resistance, culture test, GeneXpert test.
\end{abstract}

\section{Introduction}

Tuberculosis (TB) remains a major public health problem in Bangladesh. Bangladesh ranks seventh in the list of the 30 high TB burden countries. ${ }^{1}$ Accurate diagnosis and early treatment of TB has the potential to reduce morbidity and mortality associated with TB lymphadenitis (TBL). However, the differential diagnosis of TBL is broad and laboratory confirmation is the most important to guide appropriate therapy. ${ }^{2,3}$

Cytology and conventional smear microscopy have been used as the initial diagnostic tools for TBL in resource poor settings. ${ }^{3,4}$ Fine needle aspiration cytology
(FNAC) is a simple and rapid diagnostic technique, but with low specificity because of the presence of similar cytological features in lesions other than those associated with TB. ${ }^{5,6}$ Conventional Ziehl-Neelsen (ZN) stained smear microscopy lacks sensitivity due to the paucibacillary nature of fine needle aspirates (FNA). ${ }^{7}$ Mycobacteriological culture and drug susceptibility testing are not always available in resource poor settings like Bangladesh. ${ }^{1,8}$ For these limitations more rapid and reliable methods are needed. In December 2010, World Health Organization (WHO) endorsed GeneXpert MTB/RIF (Cepheid, USA) for use in TB laboratories. ${ }^{9}$ The GeneXpert assay consists

\footnotetext{
1. TE Nur, Assistant Professor of Pathology, Gazi Medical College, Khulna. Email: drtamannaenur@gmail.com

2. AU Hosna, Assistant Professor of Pathology, Prime Medical College, Rangpur

3. N Rayhan, Associate Professor of Pathology, Gazi Medical College, Khulna

4. N Nazneen, Assistant Professor of Community Medicine, Gazi Medical College, Khulna
} 
of a closed system that is based on real-time polymerase chain reaction (PCR). It can be used by operators with minimal technical expertise, enabling the diagnosis of TB and simultaneous detection of rifampicin resistance within 2 hours. $^{9}$

The GeneXpert assay has been validated and optimized for sputum samples to diagnose HIV-associated TB and multidrugresistant TB. WHO strongly recommends widespread use of GeneXpert for these groups of patients. ${ }^{10,11}$ More recently a number of studies were conducted to evaluate this assay using non-respiratory clinical samples from patients suspected of having extra-pulmonary TB (EPTB). $8,12,13$ In 2014, WHO has recommended GeneXpert over the conventional tests (including conventional microscopy, culture or histopathology) for testing specific non-respiratory specimens (lymph nodes and other tissues) from patients suspected of having EPTB. ${ }^{14}$ However, this was a conditional recommendation due to very low-quality evidence available. More studies are therefore needed particularly in settings with high EPTB prevalence. Thus, we evaluated the performance of GeneXpert for the diagnosis of TBL using routinely collected FNA material.

The purpose of this study was to test the efficiency and reliability of the GeneXpert test for the detection of MTB bacteria in suspected lymph node TB aspirated material and to compare it to conventional culture method.

\section{Materials and Method}

Ethical clearance was first obtained from the Ethical Review Board, Bangabandhu Sheikh Mujib Medical University (BSMMU). The cross sectional study was conducted in collaboration with International Centre for Diarrhoeal Disease Research Bangladesh (icddr,b).

All patients or guardians in case of children were requested for written consent prior to enrolment in the study. Any information concerning the patients was kept confidential. Laboratory results were reported back to the physicians for treatment initiation or decision as early as available.

This study was conducted at a public tertiary care hospital in Dhaka, Bangladesh, where FNAC of lymph node lesions was done in the study subjects from July 2013 to May 2015. During this period, FNAC was performed on a total of 317 clinically suspected tuberculous lymphadenitis patients without having anti-TB drugs within 60 days of sample collection, malignancies, and inadequate sample (aspirated material) drawn.

Demographic and clinical information from the subjects were collected using a pretested questionnaire. The FNA sample was collected by a resident pathologist of the Department of Pathology, BSMMU.

Gross specimen appearance (caseous, purulent, and/or blood stained) was recorded at the time of specimen collection. The first one drop of the aspirates was used for cytomorphological diagnosis. Smears were fixed in 95\% ethyl alcohol and stained with Papanicolaou staining. Another drop of specimen was used to make a smear for standard ZN staining. Both smears are examined by the same pathologist.

The cytological criteria for the diagnosis of TBL are based on the presence of the following cytomorphological appearances: epithelioid cell aggregate with or without Langhans giant cells and necrosis, epithelioid cell aggregate without necrosis, necrosis without epithelioid cell aggregate or polymorphonucleocytes with necrosis. ${ }^{15}$

The ZN stained smears were examined for the presence of AFB under oil-immersion (100x) using a light microscope. The remaining of the sample was processed for GeneXpert test conducted in the Department of Microbiology, BSMMU under the supervision of icddr,b, and for culture test done at the Tuberculosis Laboratory, icddr,b. Mycobacterial culture was done on Löwenstein-Jensen medium within 2 days of specimen collection.

Data were analyzed using the SPSS 
software package (version 17). Sensitivity, specificity, positive and negative predictive values were calculated using culture for $M$. tuberculosis as a reference standard. Statistical analysis was done by Pearson Chisquare test and a $p$ value $<0.05$ was taken as significant.

\section{Results}

The culture test revealed positive in $23.3 \%$ (Table 1). GeneXpert showed positive result in 167 patients (52.7\%). Out of these, 71 cases were culture positive while 96 were culture negative (Table 1). Sensitivity, specificity, positive predictive value and negative predictive value of GeneXpert were $95.9 \%$, $60.5 \%, 42.6 \%$ and $98.0 \%$, respectively; the percentage of agreement of GeneXpert with culture was 0.39 (Kappa test) (Table 2). GeneXpert also detected 8 RIF resistance cases.

\section{Discussion}

Based on very low quality evidence, WHO also conditionally recommends GeneXpert to be used rather than conventional methods as the initial diagnostic test in patients suspected of having EPTB. ${ }^{11,14}$ In this study, the sensitivity, specificity, positive predictive value and negative predictive value of GeneXpert test were $95.9 \%, 60.5 \%, 42.6 \%$ and $98.0 \%$, respectively. The percentage of agreement of GeneXpert test results with culture test results was 0.39 (Kappa test) implying a slight agreement. The sensitivity of GeneXpert in the current study is similar what was found in a similar study by Ligthelm et al (sensitivity $96.7 \%$ ). ${ }^{15}$ The specificity $(60.5 \%)$ of the GeneXpert in the current study was found to be consistent with a previous study reported by Biadigilegn et al (specificity $69.2 \%$ ). ${ }^{13}$ The specificity of GeneXpert test in this study was slightly lower in comparison to studies mentioned above. The presence of an unrepresentative FNA specimen, the scanty number of organisms in the lymph node lesions, nonviable organisms due to a decontamination process, and the presence of amplified false positive signals might all account for the reduced specificity. ${ }^{12,16}$

In developing countries like Bangladesh, ZN stained smear microscopy is the only widely

Table 1. Association between the results of culture test and GeneXpert test

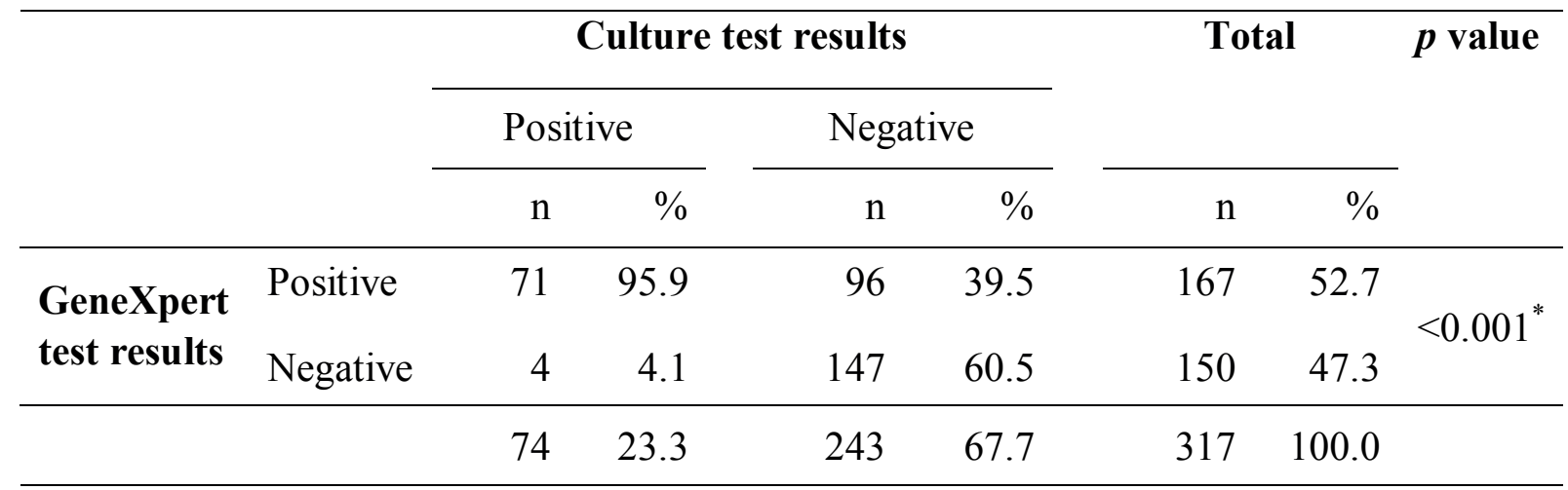

n, number. *: Pearson Chi-square test.

Table 2. Some characteristics of GeneXpert test results

\begin{tabular}{lc}
\hline Characteristics & Value \\
\hline Sensitivity & $95.9 \%$ \\
Specificity & $60.5 \%$ \\
Positive predictive value & $42.6 \%$ \\
Negative predictive value & $98.0 \%$ \\
Agreement (Kappa test) & $0.39 \%$ \\
\hline
\end{tabular}


implemented method for quantifying the bacterial burden at the time of the initial diagnosis in FNA aspirated material. GeneXpert provides a semi-quantitative measurement of the number of MTB present in a sample. In this study, more than $90 \%$ of GeneXpertpositive samples were scored as 'low' and 'very low' suggesting a limited number of bacilli in FNA sample. ${ }^{17}$

FNA cytology as an inexpensive and reliable tool for TBL has been studied by a number of investigators. ${ }^{3,18,19}$ It is one of the most commonly used methods in resource poor settings. In the current study, the sensitivity and specificity of culture test compared to that of GeneXpert, was lower. To the best of our knowledge, information regarding the drug resistance pattern of mycobacterial strains isolated from TBL patients in Bangladesh is unavailable. GeneXpert test offers rapid detection of RIF resistant MTB strains directly from the clinical sample, an important advantage over culture test. Previous studies reported $98-100 \%$ agreement in detection of RIF resistance strains using the GeneXpert test and phenotypic drug susceptibility test. ${ }^{13,14,20,21}$ In this study, GeneXpert also detected 8 RIF resistance cases.

In conclusion, our findings indicated that GeneXpert MTB/RIF test is a useful tool for the detection of MTB with high sensitivity and specificity on FNA material with superior performance as compared to culture test. Besides improved sensitivity, the GeneXpert was able to identify patients with TBL due to RIF resistant TB. The GeneXpert test is an easy and suitable method to be used in TB endemic settings and its implementation could significantly improve the rapid diagnosis of TBL.

\section{References}

1. World Health Organization (WHO). Global tuberculosis control. Geneva: WHO, 2015.

2. Kurabachew M., Enger $\varnothing$., Sandaa R. A., Skuce R., Bjorvatn B. 2004. A multiplex polymerase chain reaction assay for genus-, group- and species-specific detection of mycobacteria. Diagn Micro- biol Infect Dis 2004;49:99-104. doi: 10.1016/j.diagmicrobio.2004.03.010.

3. Wright CA, van der Burg M, Geiger D, et al. Diagnosing mycobacterial lymphadenitis in children using fine needle aspiration biopsy: cytomorphology, ZN staining and autofluorescence - making more of less. Diagn Cytopathol 2008;36:245-51. doi: 10.1002/dc.20788.

4. Kidane D, Olobo JO, Habte A, et al. Identification of the causative organism of tuberculous lymphadenitis in Ethiopia by PCR. J Clin Microbiol 2002;40:4230-4.

5. Ammari FF, Hani AHB, Ghariebeh KI. Tuberculosis of the lymph glands of the neck: a limited role for surgery. Otolaryngol Head Neck Surg 2003;128(4):576-80.

6. Boeree MJ, Kamenya A, Liomba G, Ngwira B, Subramanyam VR, Harries AD. Tuberculosis lymphadenitis, a diagnostic problem in areas of high prevalence of HIV and tuberculosis. Malawi Medical Journal 2014;11:56-9.

7. Annam V, Karigoudar MH, Yelikar BR. Improved microscopical detection of acid fast bacilli by the modified bleach method in lymph node aspirates. Indian J Pathol Microbiol 2009;52:349-52.

8. Al-Ateah SM, Al-Dowaidi MM, El-Khizzi NA. Evaluation of direct detection of Mycobacterium tuberculosis complex in respiratory and non-respiratory clinical specimens using the Cepheid Gene Xpert@ system. Saudi Med J 2012;33(10):1100-5.

9. World Health Organization (WHO). Rapid implementation of the Xpert MTB/RIF diagnostic test technical and operational 'how-to' practical considerations. Global tuberculosis control. Geneva: WHO, 2011.

10.World Health Organization (WHO). Strategic and Technical Advisory Group for Tuberculosis (STAG-TB). Report of the Tenth Meeting. Geneva: WHO, 2010.

11. World Health Organization (WHO). Automated real-time nucleic acid amplification technology for rapid and simultaneous detection of tuberculosis and rifampicin resistance: Xpert MTB/RIF assay for the diagnosis of pulmonary and extrapulmonary TB in adults and children: policy 
policy update. Geneva: WHO, 2013.

12. Hillemann D, Rüsch-Gerdes S, Boehme C, Richter E. Rapid molecular detection of extrapulmonary tuberculosis by the automated GeneXpert MTB/RIF system. J Clin Microbiol 2011;49(4):1202-5.

13.Biadglegne F, Mulu A, Rodloff AC, Sack $U$. Diagnostic performance of the Xpert MTB/RIF assay for tuberculous lymphadenitis on fine needle aspirates from Ethiopia. Tuberculosis (Edinb) 2014;94(5):502-5.

14. World Health Organization (WHO). Rapid implementation of the Xpert MTB/RIF diagnostic test technical and operational 'how-to' practical considerations. Global tuberculosis control. Geneva: WHO, 2014.

15.Ligthelm LJ, Nicol MP, Hoek KG, et al. Xpert MTB/RIF for rapid diagnosis of tuberculous lymphadenitis from fineneedle-aspiration biopsy specimens. J Clin Microbiol 2011;49:3967-70.

16. Iwnetu R, Hombergh J, Woldeamanuel Y. Is tuberculous lymphadenitis over- diagnosed in Ethiopia? Comparative performance of diagnostic tests for mycobacte- rial lymphadenitis in a high-burden country. Scand J Infect Dis 2009;41:462-8.

17.Tadesse M, Abebe G, Abdissa K, et al. GeneXpert MTB/RIF assay for the diagnosis of tuberculous lymphadenitis on concentrated fine needle aspirates in high tuberculosis burden settings. PLOS ONE 2015;10(9):e0137471.

18.Das DK. Fine needle aspiration cytology in diagnosis of tuberculous lesion. Lab Med 2000;31:625-32.

19. Mistry Y, Ninama GL, Mistry K, Rajat R, Parmar R, Godhani A. Efficacy of fine needle aspiration cytology, ZiehlNeelsen stain and culture (BACTEC) in diagnosis of tuberculous lymphadenitis. Natl J Med Res. 2012;2:77-80.

20.D. Helb, M. Jones, E. Story, et al, Rapid detection of Mycobacterium tuberculosis and rifampin resistance by use of on-demand, near-patient technology. J Clin Microbiol 2010;48:229-37.

21.Boehme CC, Nabeta P, Hillemann D, et al. Rapid molecular detection of tuberculosis and rifampin resistance. $\mathrm{N}$ Engl $\mathrm{J}$ Med 2010;363(11):1005-15.

\section{Suggestion for citation of the above:}

Nur TE, Hosna AU, Rayhan N, Nazneen N. Diagnosis of lymph node tuberculosis using the GeneXpert MTB/RIF in Bangladesh. Mediscope 2019;6(1):19-23. 